\title{
A research model for carbon-partitioning in sugarcane
}

Yellow Canopy Syndrome (YCS), first observed in 2012, is a undiagnosed condition affecting Australian sugarcane. It causes decreasing leaves to turn yellow, Frederik Botha overses YCS research for Sugar Research Australia (SRA). Focused on gene expression and protein and metabolite levels, this research seeks molecular targets to improve genetic tolerance to YCS and enhance sugarc productivity in general. In sucrose build-up regulates le metabolism through feedback control has been developed. Understanding what elevates leaf sucrose levels as YCS develops could provide insights into mechanisms underpinning sugarcane diseases and

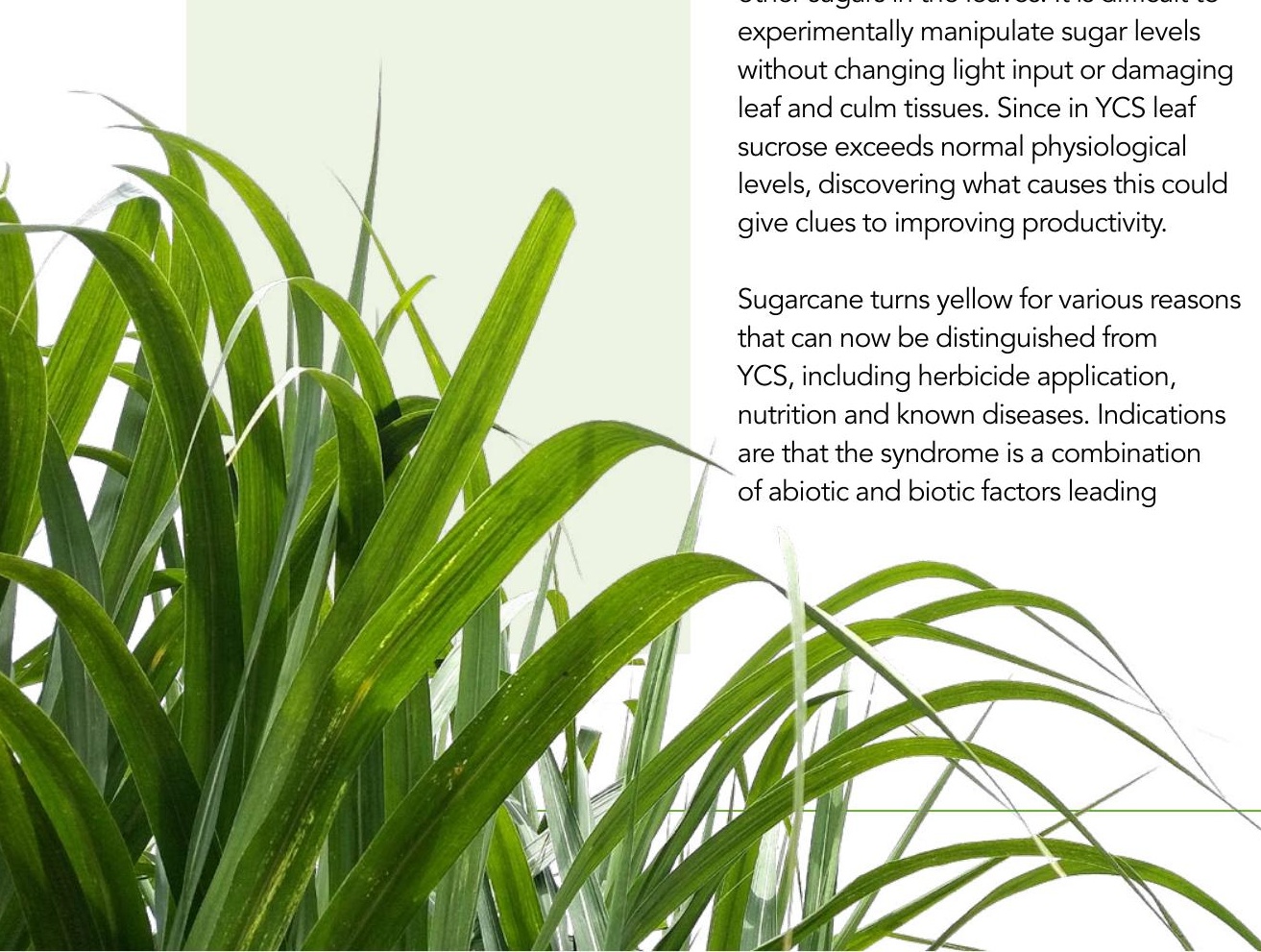

to a physiological disorder. Dr Botha and colleagues have found that YCS is especially associated with altered carbon-partsoning in the leaf. Disruption sugars to accumulate in leaves, and whe sugar exceeds a critical level it induces senescence. High levels of sucrose in sugarcane leaves are therefore an indicator of compromised crop health.

\section{THE SOURCE-SINK SYSTEM} How well a plant grows depends on acquiring raw material (carbon fixation and mineral uptake), distributing this through plant organs and coping with environmental stresses. The process known as carbon-partitioning is critical for distributing the energy captured by plants through photosynthesis. In C4 plants like sugarcane, $\mathrm{CO}_{2}$ is converted into four-carbon sugar compounds. The then enter into chemical reactions that ore

Carbon fixed during photosynthesis and converted to to sugar in 'source' cells is distributed to 'sink' cells. Phloem is the tissue that transports the soluble organic compounds (mainly sucrose),
made during photosynthesis and known as photosynthates, to wherever they are needed in the plant. The sugars are imported into sink tissues for consumption (providing energy for plant functions) or storage. Some stored sugars provide structural biomass as cellulose, emicelluloses and lignin.

Sucrose synthesis in source tissue, its translocation and in partitioning between

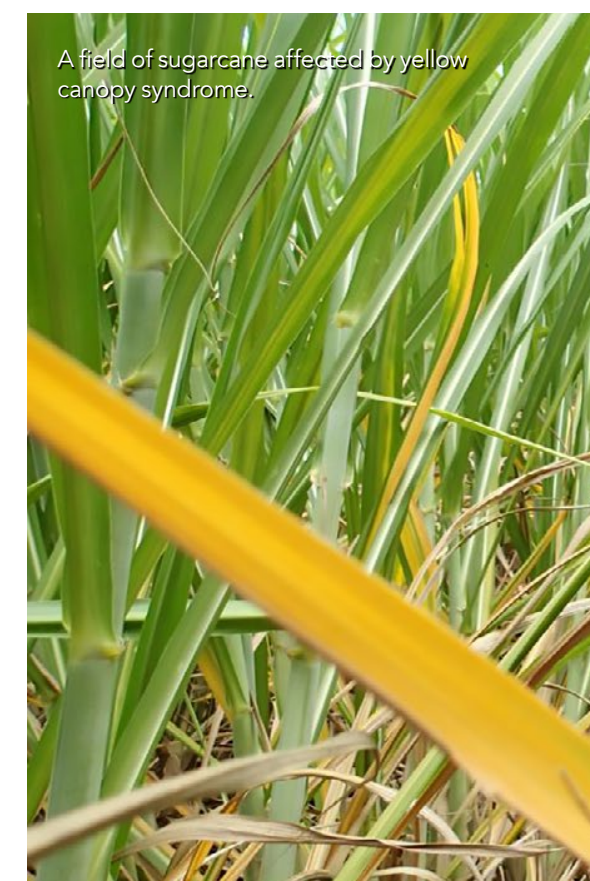

systemically coordinated in plants Not only is sucrose the priman product of photosynthesis and the building block for biomass accumulation but it also senes as a sensitive metabolic switch controlling photosynthesis and carbon-partitioning in the plant. A model for the biochemical process of carbon-partitioning in sugarcane is being developed through research on YCS.

Sugarcane has a unique source-sink tosynthates as soluble sucrose, which can reach exceptionally high concentrations in commercial sugarcane varieties. Most other plant stems store carbon as or cellulose) with low con (suctras stach sucrose. In many plants, sucrose is stored (after conversion to insoluble starch) in terminal sink orga such as tubers,

grains or fruits, rather than in the stem. Valuable sucrose from sugarcane culms is extracted and purified for use in the food industry or fermented to produce ethano.

During development, sucrose synthesised in sugarcane leaves is translocated via phloem to intemodes (he stem sections that run between leaf-carying nodes), the storage sink. Sucrose accumulates in the symplast and apoplast respectively. carbon into protein and fibre, whereas mature culms mainly partition it to sucrose storage. During maturation of commercial sugarcane cultivars, leaf photosynthe content increases, Thus, sink rege of source capacity is taking place.

\section{SUCROSE ACCUMULATION} IN SUGARCANE In YCS, leaf yellowing occurs in the late stage of sucrose accumulation, senescence is induced and tissue death begins. Normal diurnal changes morning and high at the (ow the are absent in YCS affected plonts, even Sucrose serves as a sensitive metabolic there is currently no switch controlling photosynthesis and evidence) or arise carbon-partitioning in sugarcane. $\quad \begin{aligned} & \text { not using transported } \\ & \text { sugar fast enough }\end{aligned}$ which creates an

before yellowing. So, significant metabolic overflow into the surrounding leaf blade, changes occur well before visual signs. midrib, dewlap and sheath. Increased Studies at SRA reveal that these changes sucrose also leads to elevated glucose, include an increase in soluble sugars, _ fructose and trehalose, sugars that play $\begin{array}{ll}\text { a decrease in photosynthetic rate, } & \text { major roles in metabolic signalling. } \\ \text { decreased internal leaf } \mathrm{CO}_{2} \text {, decreased } & \text { Furthermore, sucrose synthesis slows }\end{array}$ conductance through stomata (pores down which probably leads to a lowering in leaves and stems for gas exchange), of available inorganic phosphate (Pi) uncoupling of the photosynthetic within chloroplasts. A feedback signalling electron transport (PET) chain and altered mechanism involving sucrose in the 


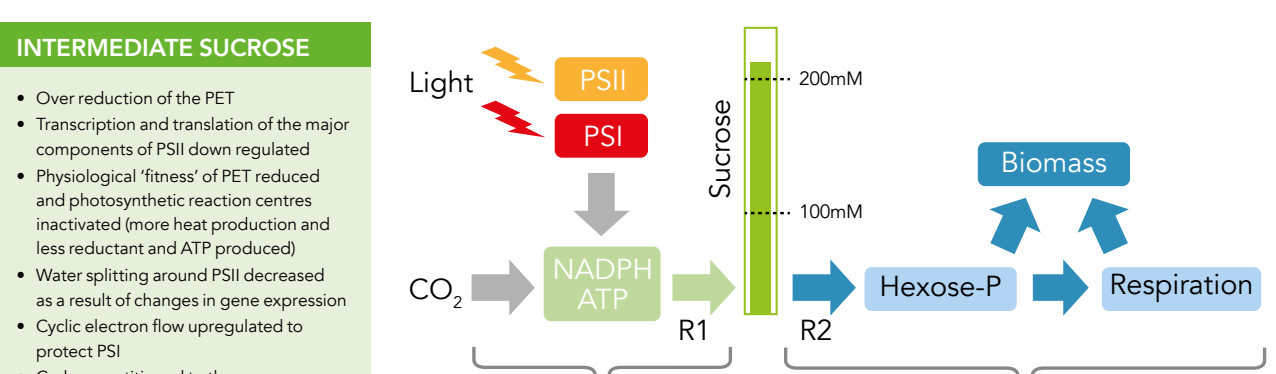

HIGH SUCROSE
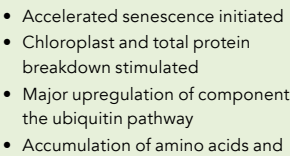

amines
Down regulation of the Ca and c3
carbon fixation patheys

te

Culm - Demand

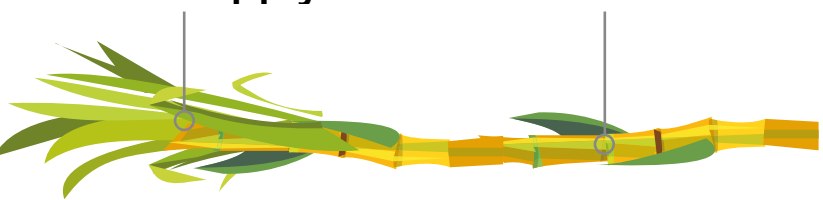

Leaf health is determined by the sucrose level in the photosynthetic mesophyll and bundle sheath cells. The sucrose level is determined by the difference between production ( $(R 1)$ and 4 tilisation in the culm ( $(22)$. Daily fluctuation between 20 and $100 \mathrm{mM}$ is normal as a result of variation in photosynthetic rate.
Between 100 and $200 \mathrm{mM}$ sucrose Between 100 and $200 \mathrm{mM}$ sucrose $(\mathrm{mitem}$ edate levels) a series of events are triggered that are aimed at protection of the photosynthetic electron transport senescence, collapse of the electron transport system, chlorophyll breakdown and cell deat.
Pi limitation. Research shows that raised photosynthetic proteins in leaf cells. From the model developed so far, YCS regulation of photosynthesis through Pi limitation leading to chronic inability to export reductant away from the PET chain during cellular sugar accumulation. Down-regulation of genes encoding Photosystem (PS) || and l, cytochrome results in decreased synthesis of these

\section{ADVANCING GENETIC STUDIES}

OF SUGARCANE

The sugarcane genome has only recently

metabolism) and transcriptome

levated sucrose. This increases caffeoy(messenger RNA molecules expressed pathways in the leaves and sink tissues of sugarcane are helping researchers to identify reactions that lead to YCS. Comparing leaf transcriptomes of symptomatic and asymptomatic plants confirms that a complex network of changes in gene expression underpin the observed changes in the metabolome.

Fluorescence and gene expression data from YCS studies indicate that PS III is the sensitive process/component, linked to reduced electron flow producing reduced co-enzyme. The early change by changes in the expression of quinic acids and quinate, compounds adical production in the chloroplast as a result of decreased electron flow to the terminal electron acceptors of PS L. Upregulation of the phenylpropanoid pathway probably shifts carbonpartitioning towards lignins, flavonoids and anthocyanins.

In the early stages of sucrose occur. significant levels of metabolites indicative of microorganisms that associate with injured tissue, especially where there are significant available in caffeoyl/chlorogenic type compounds indicative of wounding ugarcane's complexity: high polyploidy (more than two-paired sets of (varied numbers of chromosomes); bispecific origin of chromosomes; and structural differences and interspecific chromosome recombinants. A reference genome is now available for researchers. DNA sequencing, development of geneexpression technologies and improved genetic/genomics resources for Saccharum are enabling the regulatory networks of carbon-partitioning

Metabolome (low-molecular-weight metabolites produced during generation RNA sequencing to compare and CP12 (an essential regulatory proten) proteins, which then limits photosynth
A model for the biochemical defence systems; and process of carbon-partitioning increases in amino acids and metabolites indicative which is dependent on fast phosphoenolpyruvate carboxylase (PEPC). NADP-malic dehydrogenas expression is more sensitive to the This malic dehydrogenase and PEPC. This demonstrates that chloroplast metabolism is down-regulated whe sucrose levels rise.

Furthermore, genes in the shikimate and phenylpropanoid metabolic pathways regulated in early response to turnover of protein components.

A genomic approach is now being pursued for YCS in sugarcane, using nexand analyse genetic data for affected and unaffected plants from diverse field different tissue samples express differen proteins, continues to provide clues to the cause of YCS and to understanding locations. Genetic explorations of how

\section{Behind the Research}

\section{Dr Frederik (Frikkie) Botha}

E: fbotha@sugarresearch.com.au
com.au/team/drfrikkie-botha/

Research Objectives

Dr Botha's work examines leaf sucrose levels in sugarcane, among other plants, and their impact

\section{Detail}

50 Meiers Road Indooroopilly Australia

Frederik (Frikkie) Botha is the Executive Manager Strategic Initiatives at Sugar Research Australia and Honorary Professor at the University of Queensland, Australia. His research focus is on the genetic and molecular control of carbon partitioning in the culm and leaves of sugarcane, which is the driver of biomass composition and yield. The research aims to understand the control of carbon partioning between the cell wall components, respiration on suction accumulation in the culm and the impact of this on sink strength. An early switch to sucrose accumulation reduces biomass accumulation and reduces sink strength. The limited capacty to buffer leaf sucrose through partition of carbon to starch requires maintenance of a strong sink in the canopy.

\section{unding}

gar Research Australia

- Australian Research Council

- University

\section{CoAuthors}

- Annelie Marquardt (SRA) Sugar Research Australia - Gerard Scalia (SRA) Sugar Research Australia - Kate Wathen-Dunn (SRA) Sugar Research Austral Q Q Queensland Alliance for Agriculture and Food Innovation, The Univers

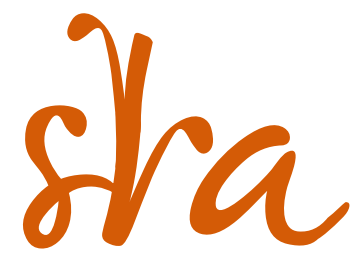

Sugar Research Australia

\section{References}

Botha, F. (2019) Metabolic Changes Associated with the Development of Yellow Canopy Syndrome in Sugarcane. Conference: International Plant and Animal Genome Conference XXIII 2015,

www.researchgate.net/publication/268116827_Metabolic_ Changes_Associated_with_the_Development_of_Yellow_ Canopy_Syndrome_in_Sugarcane

Marquardt, A., Scalia, G., Wathen-Dunn, K., Botha, F.C. (2017) Yellow Canopy Syndrome (YCS) in sugarcane is associated with altered carbon partitioning in the leaf. Sugar Tech, 19 $647-655$

Marquardt A., Scalia G., Joyce P., Basnayake J., Botha F.C. (2016) Changes in photosynthesis and carbohydrate Canopy Syndrome (YCS). Funct Plant Biol 43:523-533.

Wang. J, Nayak, S., Koch, K., Ming, R. (2013) Carbon partitioning in sugarcane (Saccharum species). Frontiers in Plant Science, 4, 201.

Sugar Research Australia. Yellow Canopy Syndrome. https:// sugarresearch.com.au/growers-and-millers/pests-and-
diseases/yellow-canopy-syndrome/ [Accessed January 2019]

\section{Personal Response}

\section{What impact do you hope this research will have over} the next five years?

II Conventional and genetic manipulation studies have penalties consistent with sucrose feedback control on photosynthesis. We need a better understanding of why sucrose accumulates in the leaves of sugarcane during stress and what the impact of this is on leaf metabolism and solutions for physiological disorders and biotic manass thent lead to sucrose accumulation. However, more importantly it could lead to genetic targets that provide an opportunity to break out of the current yield plateau that has frustrated sugarcane breeders for the past three decades. 